\title{
Abiraterone acetate treatment lowers 11-oxygenated androgens
}

\section{Connor Wright' ${ }^{1}$, Patrick O'Day', Mohammed Alyamani ${ }^{3}$, Nima Sharifi ${ }^{3,4,5}$ and Richard J Auchus ${ }^{1,2}$}

${ }^{1}$ Division of Metabolism, Diabetes, and Endocrinology, Department of Internal Medicine, ${ }^{2}$ Department of Pharmacology, University of Michigan, Ann Arbor, Michigan, USA, ${ }^{3}$ Genitourinary Malignancies Research Center, Lerner Research Institute, ${ }^{4}$ Department of Urology, Glickman Urological and Kidney Institute, and ${ }^{5}$ Department of Hematology and Oncology, Taussig Cancer Institute, Cleveland Clinic, Cleveland, Ohio, USA
Correspondence

should be addressed

to R J Auchus

Email

rauchus@med.umich.edu

\begin{abstract}
Context: The human adrenal is the dominant source of androgens in castration-resistant prostate cancer (CRPC) and classic 21-hydroxylase deficiency (21OHD). Abiraterone, derived from the prodrug abiraterone acetate (AA), inhibits the activity of cytochrome P450 17-hydroxylase/17,20-lyase (CYP17A1), the enzyme required for all androgen biosynthesis. AA treatment effectively lowers testosterone and androstenedione in 21OHD and CRPC patients. The 11-oxygenated androgens are major adrenal-derived androgens, yet little is known regarding the effects of AA administration on 11-oxygenated androgens.

Objective: To test the hypothesis that AA therapy decreases 11-oxygenated androgens.

Design: Samples were obtained from $21 \mathrm{OHD}$ or CRPC participants in AA or AA plus prednisone (AAP)-treatment studies, respectively.

Methods: We employed liquid chromatography-tandem mass spectrometry (LC-MS/MS) to measure the 11-oxygenated androgens, $11 \beta$-hydroxyandrostenedione, 11-ketoandrostenedione, 11 $\beta$-hydroxytestosterone, and 11-ketotestosterone, in plasma or serum samples from six 21OHD and six CRPC patients before and after treatment with AA or AAP, respectively.

Results: In CRPC patients, administration of AAP (1000 mg/day AA with prednisone and medical castration) lowered all four 11-oxygenated androgens to below the lower limits of quantitation $(<0.1-0.3 \mathrm{nmol} / \mathrm{L})$, equivalent to $64-94 \%$ reductions from baseline. In 21 OHD patients, administration of AA (100-250 mg/day for 6 days) reduced all 11-oxygenated androgens by on average $56-77 \%$ from baseline.

Conclusions: We conclude that AA and AAP therapies markedly reduce the production of the adrenal-derived 11-oxygenated androgens, both in patients with high (21OHD) or normal (CRPC) 11-oxygenated androgens at baseline, respectively. Reduction of 11-oxygenated androgens is an important aspect of AA and AAP pharmacology.
\end{abstract}

\section{Introduction}

Unlike the adrenals of most small mammals and other vertebrates, human adrenals express the enzyme cytochrome P450 17-hydroxylase/17,20-lyase (CYP17A1) and therefore produce not only cortisol but also 19-carbon steroids (androgens and androgen precursors). The zona reticularis (ZR) maintains high ACTH-dependent (c) 2020 European Society of Endocrinology Printed in Great Britain expression of CYP17A1 and the regulatory protein cytochrome $b_{5}$, which are required to efficiently convert pregnenolone to dehydroepiandrosterone (DHEA) (1). The adrenal exports large amounts of DHEA as the sulfate (DHEAS), and circulating DHEAS concentrations exceed those of all other steroids by up to several 
orders of magnitude throughout most of adult life. The

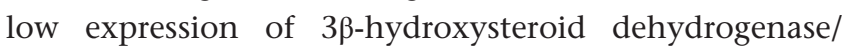
isomerase (3ßHSD) in the ZR limits conversion of DHEA to active androgens, yet the human adrenal directly produces moderate amounts of androstenedione (A4) and very small amounts of testosterone (T) (2) (Fig. 1).

While the testes are the primary source of the androgen $\mathrm{T}$ in normal adult men, the adrenals produce other 19-carbon steroids, and adrenal-derived androgens (active androgens either directly produced from the adrenal or generated in peripheral tissues from precursors synthesized in the adrenals) are a significant component in many scenarios. In prepubertal children, the expansion of the ZR and rise of adrenal-derived androgens manifest as the development of axillary and pubic hair during the process of adrenarche (3). Genetic defects in cortisol biosynthesis, collectively known as congenital adrenal hyperplasia (CAH), feature androgen excess in most forms, including 21-hydroxylase deficiency (21OHD, the most common), 11ß-hydroxylase deficiency, and

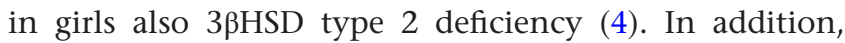
the standard initial treatment for men with metastatic prostate cancer is androgen deprivation therapy (ADT) with medical castration (5). While ADT eliminates testicular T production, adrenal-derived androgens persist and precipitate treatment failure upon transition to castration-resistant prostate cancer (CRPC) (6), which has been difficult to treat and is often fatal.

In both 21OHD and CRPC, strategies to lower adrenalderived androgens have been developed. In 21OHD, supraphysiologic glucocorticoid dosing has been a successful strategy to lower ACTH and adrenal 19-carbon steroid production from high to normal (7). In CRPC, reduction or blockade of adrenal-derived androgens has become an effective treatment strategy, but the degree of suppression or antagonism must be essentially complete.

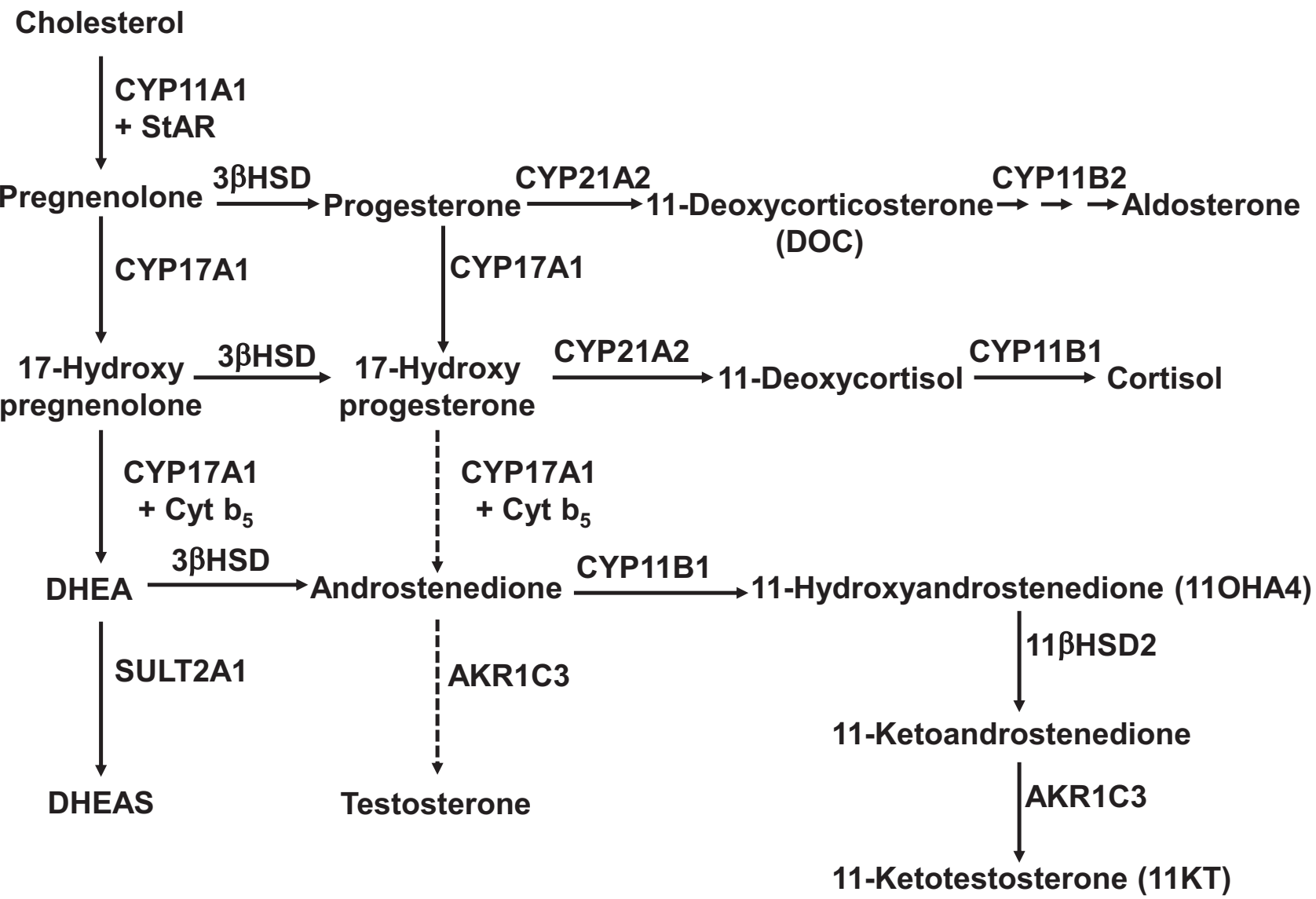

Figure 1

Abbreviated pathways of adrenal steroid biosynthesis. DHEA is primarily sulfated by sulfotransferase 2A1 (SULT2A1) to DHEAS, but a fraction is converted to A4. A4 is a good substrate for CYP11B1, yielding 11OHA4, and 11OHA4 is metabolized primarily in peripheral tissues to $11 \mathrm{KT}$. Abbreviations not in text: 11 $\beta \mathrm{HSD} 2,11 \beta$-hydroxysteroid dehydrogenase type 2; AKR1C3, aldo-keto reductase $1 \mathrm{C} 3(17 \beta \mathrm{HSD} 5)$; Cyt $b_{5}$, cytochrome $b_{5}$; StAR, steroidogenic acute regulatory protein. 
Besides third-generation anti-androgens, CYP17A1 inhibitors have become a mainstay of CRPC treatment (8). Abiraterone acetate (AA), the prodrug of abiraterone, in combination with prednisone has been shown to improve survival in CRPC patients $(9,10)$ as well as when added to ADT prior to the development of CRPC $(11,12)$. Using highly sensitive liquid chromatography-tandem mass spectrometry (LC-MS/MS) assays, 1000 mg/day AA plus prednisone $5 \mathrm{mg}$ twice daily (AAP therapy) lowers the traces of circulating $\mathrm{T}$ in most men with CRPC from $\sim 0.3$ $\mathrm{nmol} / \mathrm{L}$ to $<0.01 \mathrm{nmol} / \mathrm{L}$ (13). In 2014, we repurposed AA for the treatment of classic 21OHD in combination with physiologic hydrocortisone. The six enrolled participants received 100 or $250 \mathrm{mg}$ of AA each morning plus $20 \mathrm{mg} /$ day hydrocortisone and fludrocortisone acetate for 6 days. Using LC-MS/MS, the study found that AA at $250 \mathrm{mg} /$ day normalized A4 and T on day 7 in all participants (14).

Beyond the traditional 19-carbon steroids DHEA, A4, and $\mathrm{T}$, the human adrenal produces substantial amounts of 11-oxygenated androgens (Fig. 1). Of these, the major direct adrenal product is $11 \beta$-hydroxyandrostenedione (11OHA4) (2) via 11 $\beta$-hydroxylation of A4, and 11-ketotestosterone $(11 \mathrm{KT})$ is the major active androgen derived from 11OHA4 through peripheral metabolism (15). Although $11 \mathrm{KT}$ has been long recognized as the primary active androgen in bony fishes and 11OHA4 was identified in human serum decades ago, a broader significance of 11-oxygenated androgens in human beings has only recently emerged (16). We have shown that $11 \mathrm{KT}$ is the dominant circulating androgen during normal and premature adrenarche (15), in women and men with poorly controlled 21OHD (17), and in postmenopausal women (18). Other studies suggest that circulating $11 \mathrm{KT}$ concentrations exceed those of $\mathrm{T}$ in most women, particularly those with polycystic ovary syndrome (19), and that 11-oxygenated androgens contribute to the progression of CRPC (20). While AA has been shown to lower A4 and T in both 21OHD (from high to normal) (14) and CRPC (from normal or low to undetectable) (13), the changes in 11-oxygenated androgens were not previously assessed in AA studies. The purpose of this study is to determine the effects of AA administration on the 11-oxygenated androgens $11 \mathrm{OHA} 4,11 \mathrm{KT}$, as well as 11-ketoandrostenedione (11KA4) and 11 $\beta$-hydroxytestosterone (11OHT), to broaden our understanding of AA pharmacology.

\section{Methods}

AA was administered to the $210 H D$ patients after the first-morning dose of hydrocortisone on days 1 through 6, and plasma samples from were collected at $0800 \mathrm{~h}$ prior to all medications on days $1,6,7$, and 8 (NCT01495910). Baseline samples reflected the effect of hydrocortisone $20 \mathrm{mg} /$ day in 2-3 divided doses prior to two dose-escalated AA treatment periods, 100 and $250 \mathrm{mg} /$ day, after which the primary endpoint was met (14). The serum A4 at baseline exceeded 1.5 times the upper limit of normal in all participants (>12 nmol/L). Serum samples were collected from six CRPC patients who received AAP treatment between 09/03/2015 and 03/23/2017. Patients with metastatic CRPC were recruited through the Department of Hematology and Oncology, Taussig Cancer Institute at the Cleveland Clinic. The protocol (The Origins of Resistance to Hormonal Therapy in Advanced Prostate Cancer, case 7813) was approved by the institutional review board, and all subjects provided written informed consent. To qualify, subjects had not received prior ketoconazole treatment or chemotherapy or were currently treated with $5 \alpha$-reductase inhibitors. Serum samples were collected and banked for research purposes during standard-of-care treatment with AAP, and all six available pairs of pre- and post-AAP samples were used. Two blood samples were collected from each patient - prior to starting AAP treatment and following at least 4 weeks of treatment between 2 and $15 \mathrm{~h}$ after the 1000 mg daily dose of AA. Samples were collected in Vacutainer Plus serum blood collection tubes (\#BD367814, Becton Dickenson, Franklin Lakes, NJ) and allowed to clot. Tubes were centrifuged at $1430 \boldsymbol{g}$ for $10 \mathrm{~min}$. Serum aliquots were frozen at $-80^{\circ} \mathrm{C}$ until processing. The studies were approved by the institutional review boards of the University of Michigan or Cleveland Clinic, and all participants gave written informed consent.

Serum or plasma $(0.1 \mathrm{~mL})$ was combined with internal standards (Table 1), diluted with water, and loaded onto a supported liquid extraction cartridge (Isolute, Biotage, Charlotte, NC). Steroids were eluted twice with $0.7 \mathrm{~mL}$ methyl-tert-butyl ether, dried under vacuum (Savant, Thermo Fisher), and reconstituted in 0.1 mL 40:60 methanol:water. LC-MS/MS was performed as previously described (18), with the following modifications. Steroids were quantified with an Agilent 6495 triple quadrupole mass spectrometer and Agilent 1260 and 1290 dual front-end HPLC/UPLC equipped with a $3 \times 10 \mathrm{~mm} 5-\mu \mathrm{m}$ particle size $\mathrm{C}_{4}$ HypersilGOLD cartridge (Thermo Fisher) and a Kinetex $2.1 \times 150 \mathrm{~mm} 2.6-\mu \mathrm{m}$ particle size biphenyl resolving column (Phenomenex, Torrance, CA) in electrospray ionization mode. Mobile phases consisted of $0.2 \mathrm{mmol} / \mathrm{L}$ aqueous ammonium fluoride (phase A) and methanol with $0.2 \mathrm{mmol} / \mathrm{L}$ ammonium fluoride (phase B). 
Table 1 List of steroid analytes, standards, internal standards, and mass spectrometry parameters.

\begin{tabular}{l} 
Steroid \\
\hline Progesterone \\
Progesterone-d9 \\
17-Hydroxyprogesterone \\
17-Hydroxyprogesterone-d8 \\
Corticosterone \\
Corticosterone-d8 \\
11-Deoxycorticosterone \\
11-Deoxycorticosterone-d8 \\
11-Deoxycortisol \\
11-Deoxycortisol-d5 \\
Cortisol \\
Cortisol-d4 \\
Cortisone \\
Cortisone-d8 \\
Androstenedione \\
Androstenedione-d7 \\
Testosterone \\
Testosterone-d3 \\
11-Hydroxyandrostenedione \\
11-Hydroxyandrostenedione-d3 \\
11-Ketoandrostenedione \\
11-Hydroxyandrostenedione-d3 \\
11-Hydroxytestosterone \\
11-Ketotestosterone-d3 \\
11-Ketotestosterone \\
11-Ketotestosterone-d3 \\
\hline
\end{tabular}

\begin{tabular}{l} 
Source \\
\hline Cerilliant \\
C/D/N Isotopes \\
Cerilliant \\
C/D/N Isotopes \\
Cerilliant \\
C/D/N Isotopes \\
Cerilliant \\
Sigma-Aldrich \\
Cerilliant \\
Sigma-Aldrich \\
Cerilliant \\
Cerilliant \\
Cerilliant \\
Cerilliant \\
Cerilliant \\
Cambridge Isotopes \\
Cerilliant \\
C/D/N Isotopes \\
Steraloids \\
RTI \\
Steraloids \\
RTI \\
Steraloids \\
RTI \\
Steraloids \\
RTI \\
\hline
\end{tabular}

\begin{tabular}{llc}
\hline MRM $(\mathrm{m} / \mathrm{z})$ & & Qual $(\mathrm{m} / \mathrm{z})$ \\
\cline { 1 - 1 } $315.2 / 97.0$ & & 109.1 \\
$324.2 / 97.0$ & & 113.1 \\
$331.2 / 97.0$ & & 313.0 \\
$339.2 / 100.0$ & & 113.0 \\
$347.2 / 121.1$ & & 329.2 \\
$355.2 / 337.1$ & & 125.1 \\
$331.2 / 97.0$ & & 109.0 \\
$339.2 / 100.0$ & & 113.0 \\
$347.2 / 97.0$ & & 109.0 \\
$352.2 / 100.1$ & & 113.1 \\
$363.2 / 121.1$ & & 327.3 \\
$367.2 / 121.1$ & & 97.0 \\
$361.2 / 163.0$ & & 121.1 \\
$369.0 / 169.0$ & & 120.0 \\
$287.0 / 97.0$ & & 109.0 \\
$294.0 / 100.0$ & & 113.0 \\
$289.2 / 97.0$ & & 109.0 \\
$292.0 / 97.0$ & & 109.0 \\
$303.2 / 120.9$ & & 285.1 \\
$306.2 / 288.2$ & & 122.1 \\
$301.2 / 257.0$ & 120.9 \\
$306.2 / 288.2$ & 122.1 \\
$305.3 / 269.0$ & 105.0 \\
$308.3 / 262.3$ & & 123.1 \\
$303.2 / 120.9$ & & 259.1 \\
$308.3 / 262.3$ & & 123.1 \\
\hline & & \\
\hline
\end{tabular}

\begin{tabular}{c}
\hline $\mathbf{R T}(\mathrm{min})$ \\
\hline 14.4 \\
14.4 \\
11.4 \\
11.3 \\
10.6 \\
10.5 \\
13.0 \\
13.0 \\
10.0 \\
9.9 \\
7.3 \\
7.3 \\
8.0 \\
7.9 \\
13.0 \\
13.0 \\
11.3 \\
11.3 \\
10.5 \\
10.4 \\
10.7 \\
10.4 \\
10.5 \\
9.3 \\
9.3 \\
9.3 \\
\hline
\end{tabular}

\begin{tabular}{l}
\hline LLOQ $(\mathrm{nmol} / \mathrm{L})$ \\
\hline 0.09 \\
0.1 \\
0.6 \\
0.06 \\
0.01 \\
0.6 \\
0.7 \\
0.03 \\
0.02 \\
0.3 \\
0.1 or 265.1 \\
0.1 \\
0.1 \\
\end{tabular}

LLOQ, lower limit of quantitation (extrapolated signal-to-noise ratio of 3); $\mathrm{m} / \mathrm{z}$, mass to charge ratio; MRM, precursor and product ions for mass ratio monitoring; Qual, qualifier ion; RT, retention time; RTI, The National Heart. Lung and Blood Institute RTI International metabolite Standards Synthesis Center.

Samples were first loaded onto the $\mathrm{C}_{4}$ cartridge with isocratic $35 \%$ mobile phase $\mathrm{B}$ at a flow of $0.2 \mathrm{~mL} / \mathrm{min}$ using the 1260 HPLC pumps, and the early eluting polar matrix elements were routed to waste. Immediately prior to the elution of the first compound of interest from the $\mathrm{C}_{4}$ cartridge, a 10-port valve routed the flow from the 1290 UPLC pumps through the $\mathrm{C}_{4}$ cartridge to the biphenyl column in serial configuration, and mobile phase B was stepped to $55 \%$ to expedite elution of steroids from the $\mathrm{C}_{4}$ cartridge to the biphenyl column. After the last compound of interest was eluted from the $\mathrm{C}_{4}$ cartridge, the 10-port valve routed the flow from the 1260 HPLC pumps through the $\mathrm{C}_{4}$ cartridge to waste for washing, which eliminated many non-polar interfering matrix elements and prepared the cartridge for the next injection. Simultaneously, the 10-port valve directed the flow from the 1290 UPLC pumps directly through the biphenyl column first to waste and then from 3.01 to 14.9 min into the source of the Agilent 6495 triple quadrupole tandem mass spectrometer using a methanol/ water gradient (Table 2). The steroids were quantitated using dynamic multiple reaction monitoring (DMRM) mode (Table 1).
The lower limit of quantitation (LLOQ, Table 1) for each analyte was estimated from the signal-to-noise ratio of pooled samples, using the Mass Hunter 'peak to peak' method in a noise region window lacking interfering peaks of 0.25 min most often post-peak, and extrapolated to a concentration that yields a signal-to-noise ratio of 3 . Review of extracted ion chromatograms confirmed that all samples with values reported above the LLOQ gave reliably quantifiable peaks at the correct retention time. Baseline versus AA or AAP values were compared with twotailed paired Wilcoxon signed rank tests setting $\alpha=0.05$.

\section{Results}

\section{CRPC patients}

For the six CRPC patients, baseline 11OHA4 concentrations ranged $2-5 \mathrm{nmol} / \mathrm{L}$ and $11 \mathrm{KT} 0.4-1.4 \mathrm{nmol} / \mathrm{L}$, with $11 \mathrm{OHT}$ and 11KA4 values lower. After treatment with $1000 \mathrm{mg}$ AA daily plus prednisone $5 \mathrm{mg}$ twice daily, the active androgens 11OHT and 11KT (15) were both suppressed to below the LLOQ $(0.1 \mathrm{nmol} / \mathrm{L})$, a $73-93 \%$ reduction from baseline ( $P=0.03$, Fig. 2). Similarly, 11KA4 and 
Table 2 Chromatography scheme for LC-MS/MS.

\begin{tabular}{|c|c|c|c|c|}
\hline Time (min) & $\%$ A & $\%$ B & Routing & Flow $(\mathrm{mL} / \mathrm{min})$ \\
\hline \multicolumn{5}{|c|}{$\overline{1260 \mathrm{HPLC}} \overline{\mathrm{Pump}} 1$} \\
\hline 0.00 & 65 & 35 & $1 \rightarrow$ Waste & 0.20 \\
\hline 0.70 & 65 & 35 & $1 \rightarrow$ Waste & 0.20 \\
\hline 0.71 & 65 & 35 & Waste & 0.20 \\
\hline 0.80 & 65 & 35 & Waste & 0.20 \\
\hline 0.85 & 0 & 100 & Waste & 0.20 \\
\hline 1.20 & 0 & 100 & Waste & 0.50 \\
\hline 2.50 & 0 & 100 & Waste & 0.50 \\
\hline 2.51 & 0 & 100 & Waste & 0.10 \\
\hline 3.01 & 0 & 100 & $1 \rightarrow$ Waste & 0.10 \\
\hline 3.90 & 0 & 100 & $1 \rightarrow$ Waste & 0.10 \\
\hline 3.91 & 0 & 100 & $1 \rightarrow$ Waste & 0.10 \\
\hline 7.40 & 0 & 100 & $1 \rightarrow$ Waste & 0.10 \\
\hline 10.00 & 0 & 100 & $1 \rightarrow$ Waste & 0.20 \\
\hline 10.01 & 65 & 35 & $1 \rightarrow$ Waste & 0.50 \\
\hline 12.00 & 65 & 35 & $1 \rightarrow$ Waste & 0.50 \\
\hline 12.10 & 65 & 35 & $1 \rightarrow$ Waste & 0.20 \\
\hline \multicolumn{5}{|c|}{1290 UPLC Pump 2} \\
\hline 0.00 & 65 & 35 & $2 \rightarrow$ Waste & 0.20 \\
\hline 0.70 & 65 & 35 & $2 \rightarrow$ Waste & 0.20 \\
\hline 0.71 & 65 & 35 & $1 \rightarrow 2 \rightarrow$ Waste & 0.20 \\
\hline 0.80 & 45 & 55 & $1 \rightarrow 2 \rightarrow$ Waste & 0.20 \\
\hline 3.01 & 45 & 55 & $2 \rightarrow \mathrm{MS}$ & 0.20 \\
\hline 12.50 & 6.6 & 93.4 & $2 \rightarrow \mathrm{MS}$ & 0.20 \\
\hline 12.51 & 5 & 95 & $2 \rightarrow \mathrm{MS}$ & 0.20 \\
\hline 14.90 & 0 & 100 & $2 \rightarrow \mathrm{MS}$ & 0.25 \\
\hline 15.00 & 0 & 100 & $2 \rightarrow$ Waste & 0.25 \\
\hline 15.20 & 0 & 100 & $2 \rightarrow$ Waste & 0.50 \\
\hline 16.20 & 0 & 100 & $2 \rightarrow$ Waste & 0.50 \\
\hline 16.21 & 65 & 35 & $2 \rightarrow$ Waste & 0.40 \\
\hline 19.21 & 65 & 35 & $2 \rightarrow$ Waste & 0.40 \\
\hline
\end{tabular}

11OHA4 decreased to below LLOQ ( 0.1 or $0.3 \mathrm{nmol} / \mathrm{L}$, respectively) in all patients, which are $64-94 \%$ reductions from baseline $(P=0.03)$. For comparison, AAP therapy also lowered A4 69-90\% from baseline and T to below LLOQ $(0.02 \mathrm{nmol} / \mathrm{L})$ in all patients $(P=0.03$, Fig. 2$)$. These data indicate that AAP therapy markedly lowers 11-oxygenated androgens in CRPC patients.

\section{HD patients}

At baseline prior to the first treatment period, the six 21 OHD patients had baseline A4 and T values well above the normal range for women. We confirmed that, as we showed previously (14), AA treatment reduced A4 and T into the normal range by day 6-7 in half or all women at $100 \mathrm{mg} /$ day or $250 \mathrm{mg} /$ day, respectively ( $P=0.03$, Fig. 3). Similarly, the 11-oxygenated androgens fell markedly, particularly in those with the highest baseline values, and did not return to baseline by day 8 . With $250 \mathrm{mg} /$ day AA, 11OHA decreased
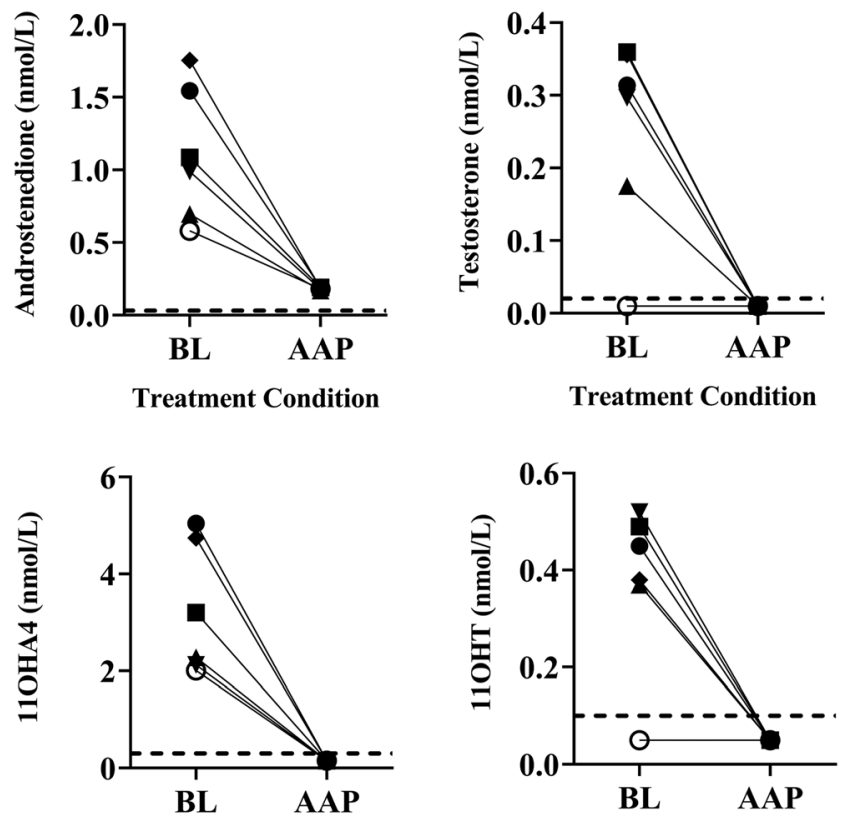

Treatment Condition
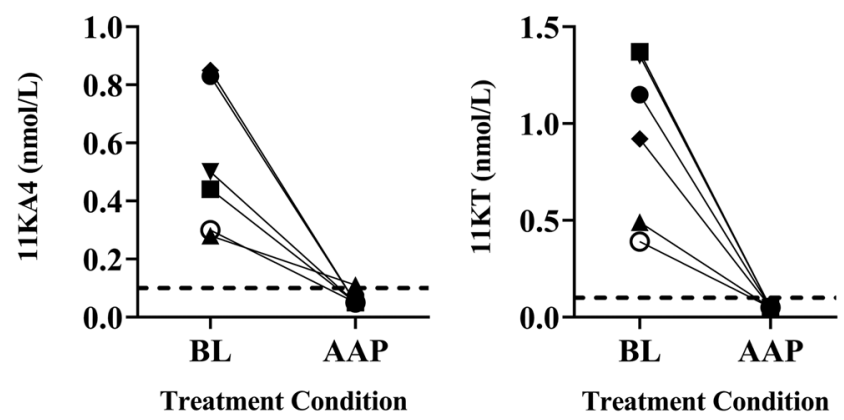

\section{Figure 2}

Reductions in conventional and 11-oxygenated androgens in CRPC patients with AAP treatment. Symbols show individual patient data from baseline (BL) and after at least 4 weeks of AAP therapy. The dotted lines indicate LLOQ for each steroid, and values $\angle L L O Q$ are plotted midway between 0 and LLOQ. Conversion factors from $\mathrm{nmol} / \mathrm{L}$ to $\mathrm{ng} / \mathrm{dL}$ : multiply value by 28.64 for A4, 28.84 for T, 30.24 for $11 \mathrm{OHA} 4$ and $11 \mathrm{KT}, 30.04$ for $11 \mathrm{KA} 4$, and 30.44 for $11 \mathrm{OHT}$.

64-98\%, 11KA decreased 63-98\%, 11OHT decreased 51-90\%, and 11KT decreased 55-97\% ( $P=0.03$, Fig. 3).

\section{Progesterone and 11-deoxycorticosterone changes}

Because administration of AA inhibits both 17-hydroxylase and 17,20-lyase activities of CYP17A1, 17-deoxysteroids upstream of androgens rise, and glucocorticoid administration attenuates this rise $(21,22)$. In patients with $21 \mathrm{OHD}$, the cytochromes P450 CYP3A4 


\section{Period 1}
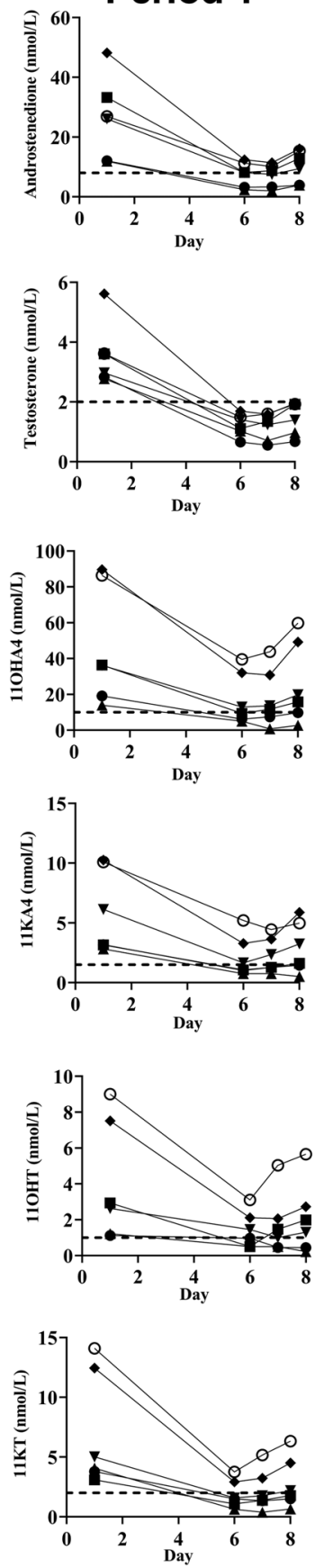
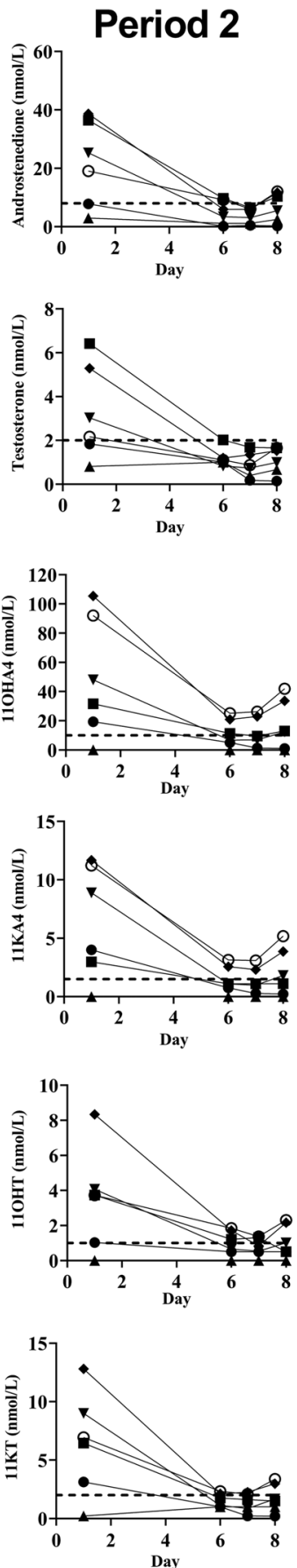

Figure 3

Changes in conventional and 11-oxygenated androgens with AA in patients with $210 \mathrm{HD}$. Symbols show individual patient data from baseline (day 1 ) and days $6-8$ for 6 days of AA therapy at $100 \mathrm{mg} /$ day (Period 1) or $250 \mathrm{mg} /$ day (Period 2).

Dotted lines indicate upper limit of normal ranges for premenopausal women (18). Conversion factors from nmol/L to $\mathrm{ng} / \mathrm{dL}$ : multiply value by 28.64 for $\mathrm{A} 4,28.84$ for $\mathrm{T}, 30.24$ for $11 \mathrm{OHA} 4$ and $11 \mathrm{KT}, 30.04$ for $11 \mathrm{KA} 4$, and 30.44 for $11 \mathrm{OHT}$.

and CYP2C19 in the liver 21-hydroxylate circulating progesterone to 11-deoxycorticosterone (DOC) (23). We therefore also sought to assess changes in progesterone and DOC in patients with $210 \mathrm{HD}$ during AA treatment.

In the $21 \mathrm{OHD}$ patients, median progesterone increased as anticipated by $148 \%$ in period 1 and by $114 \%$ in period 2 on day 6; however, proportionate increases in DOC did not accompany these increases in progesterone. In period 1 , median DOC rose only $3 \%$, and in period 2 , DOC did not change. Consequently, the median progesterone/ DOC ratio increased by $121 \%$ in period 1 and by $106 \%$ in period $2(P=0.03$, Fig. 4$)$.
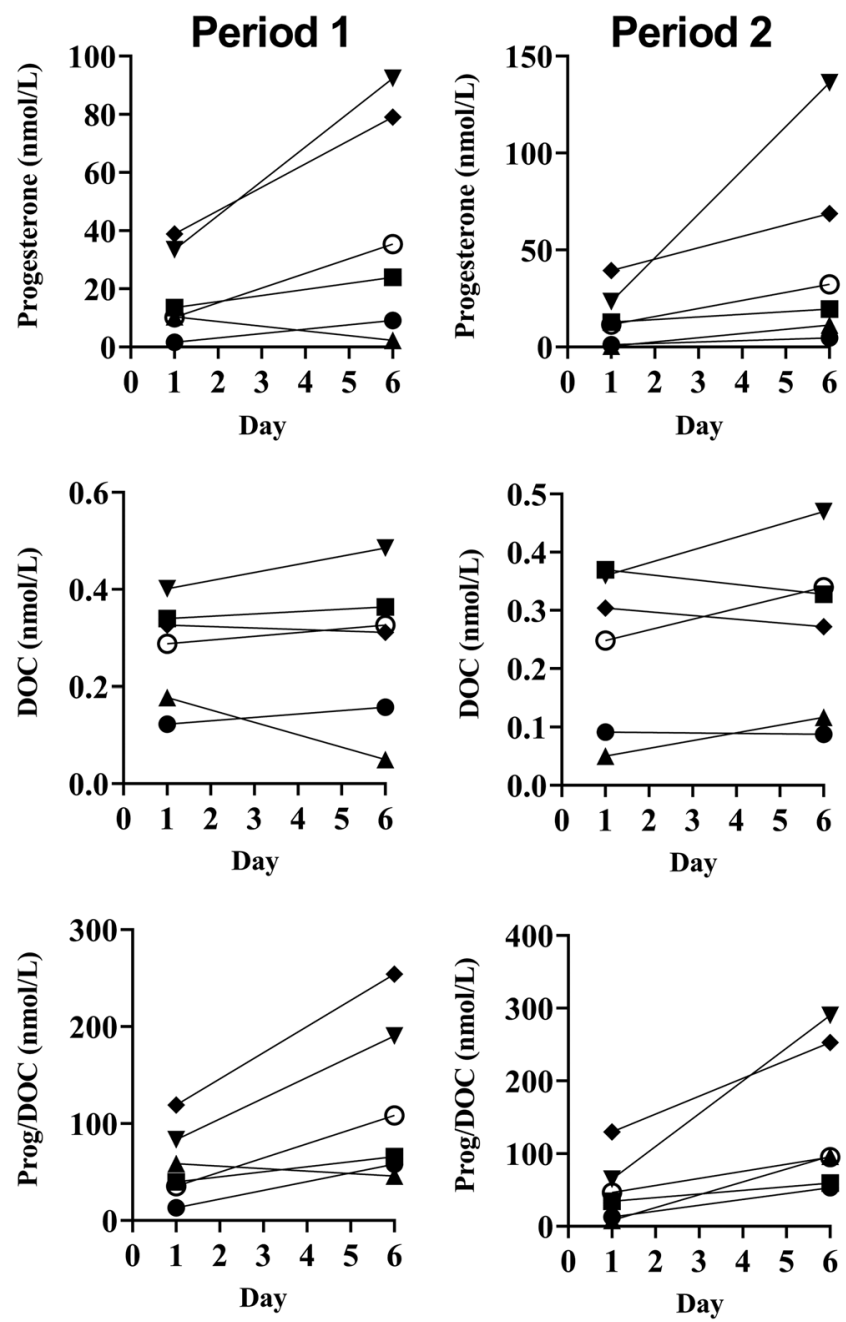

Figure 4

Changes in progesterone, DOC, and progesterone/DOC (Prog/ DOC) ratio in $210 \mathrm{HD}$ patients with AA treatment. Symbols show individual patient data from baseline and after 6 days of AA therapy at $100 \mathrm{mg} /$ day (Period 1) or $250 \mathrm{mg} /$ day (Period 2). Conversion factors for ng/dL: multiply value by 31.45 for progesterone and 33.05 for DOC. 

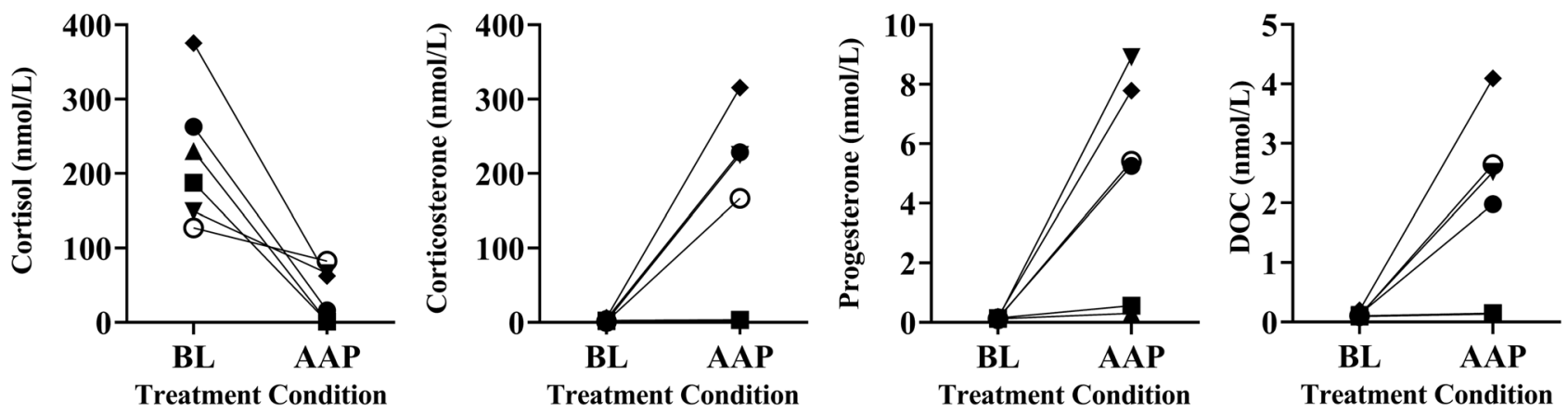

\section{Figure $\mathbf{5}$}

Changes in adrenal 21-carbon steroids from CRPC patients at baseline and with AAP treatment. Symbols show individual patient data from baseline and after at least 4 weeks of AAP therapy. Conversion factors for ng/dL: multiply by 36.25 for cortisol, 34.65 for corticosterone, 31.45 for progesterone, and 33.05 for DOC.

Unlike the 21OHD patients, the CRPC patients experienced a 20-fold rise in median DOC (0.11 to $2.25 \mathrm{nmol} / \mathrm{L})$. This rise in DOC was accompanied by substantial rises in median corticosterone (2.25 to 195.6 $\mathrm{nmol} / \mathrm{L})$ and progesterone (0.13 to $5.33 \mathrm{nmol} / \mathrm{L})(P=0.03$ for all changes, Fig. 5). Median cortisol decreased by $81 \%$ from baseline, yet this adrenal axis suppression was variable across the six patients. In patients 4,5 , and 6 , on-treatment cortisol ranged $62.6-82.6 \mathrm{nmol} / \mathrm{L}$, while in patients 1, 2, and 3, treatment cortisol ranged 1.0-16.1 nmol/L (Fig. 5).

\section{Discussion}

The major finding of this study is that AA and AAP therapies effectively and consistently lower 11-oxygenated androgens in both $210 \mathrm{OD}$ and CRPC patients, respectively, in parallel with conventional androgens A4 and T. AA or AAP therapies were effective in lowering 11-oxygenated androgens from high values (up to ten times the upper limit of normal) to within or much closer to normal in 21 OHD patients and from normal values to below LLOQ in CRPC patients, illustrating efficacy in two very different conditions. Although the cohorts consisted of only six patients each, the results were consistent and dramatic across all patients in both cohorts, and the results were replicated in a second treatment period for the $210 H D$ patients. Consequently, we report these results with confidence.

Our data support the hypothesis that AA therapy lowers 11-oxygenated androgens. The 11-oxygenated androgens are now recognized as the major androgens in patients with $210 H D(17)$, and new strategies to lower
A4 and T $(14,24,25,26)$ must also reduce 11-oxygenated androgens, as we now show for AA. As observed for A4 and $\mathrm{T}$ in the 21OHD study (14), 11-oxygenated androgens remained below baseline on day $8,48 \mathrm{~h}$ after the last AA dose, consistent with extremely tight binding of abiraterone to CYP17A1 (27). Although 11-oxygenated androgens were previously neglected in CRPC, prostate cancer cell lines contain enzymes to convert 11OHA4 to active androgens $(28,29,30)$, and 11-oxygenated androgens promote growth of these cells (20). Our data suggest that inhibition of 11-oxygenated androgen production might significantly contribute to the efficacy of AAP in the treatment of CRPC, and direct inhibition of adrenal CYP17A1 and 11-oxygenated androgen synthesis, which is not achieved with ADT alone, might contribute to the efficacy of AAP as initial treatment of metastatic prostate cancer.

Complete or partial aldosterone deficiency contributes to the propensity for infants and children with 21OHD to experience adrenal crises, yet hypertension is observed in some adults with $21 \mathrm{OHD}$ (7). One potential mechanism of this phenomenon is the extra-adrenal 21-hydroxylation of circulating adrenal-derived progesterone to DOC via hepatic P450 enzymes (23), as is observed in normal pregnancy with placenta-derived progesterone (31). In one study of patients with classic 21OHD, DOC did not rise with cosyntropin stimulation (32), consistent with extra-adrenal genesis. Alternatively, human CYP17A1 has a small amount of progesterone 21-hydroxylase activity, which is accentuated with $\left(16 \alpha, 17-{ }^{2} \mathrm{H}_{2}\right)$-progesterone substrate, a consequence of metabolic switching due to intramolecular kinetic isotope effects (33). If DOC production in $210 H D$ was solely due to hepatic metabolism of circulating progesterone, the AA therapy, which raises 
progesterone, should also raise DOC proportionately, but if CYP17A1-catalyzed the DOC formation, AA should lower DOC production. Our observation that DOC remains relatively stable with AA treatment for $210 H D$ despite a $>2$-fold increase in progesterone suggests that a portion of DOC derives from both pathways.

We conclude that AA decreases adrenal-derived 11-oxygenated androgens in patients with either very low or very high androgens at baseline. This action of AA is likely to contribute significantly to the efficacy of AAP in the treatment of patients with CRPC and of AA in the treatment of patients with 21OHD.

\section{Declaration of interest}

$\mathrm{RJ} A$ is a consultant for Janssen Pharmaceuticals. The other authors have nothing to disclose.

\section{Funding}

This work was supported by Janssen Research and Development and Grant UL1-RR024986 from the National Institutes of Health to the Nursing and Laboratory Staff of the Michigan Clinical Research Unit. The authors also thank Janssen for providing samples obtained from the study (NCT01495910) for this research. R J A received support from Grant R01GM086956 from the National Institutes of Health. C W participated in the University of Michigan Pharmacology Summer Undergraduate Research Program. This work also received support, in part, from the National Cancer Institute (R01CA172382 and R01CA190289) and the Prostate Cancer Foundation (to N S).

\section{References}

1 Auchus RJ, Lee TC \& Miller WL. Cytochrome $b_{5}$ augments the 17,20 lyase activity of human P450c17 without direct electron transfer. Journal of Biological Chemistry 1998273 3158-3165. (https://doi. org/10.1074/jbc.273.6.3158)

2 Rege J, Nakamura Y, Satoh F, Morimoto R, Kennedy MR, Layman LC, Honma S, Sasano H \& Rainey WE. Liquid chromatography-tandem mass spectrometry analysis of human adrenal vein 19-carbon steroids before and after ACTH stimulation. Journal of Clinical Endocrinology and Metabolism 201398 1182-1188. (https://doi. org/10.1210/jc.2012-2912)

3 Auchus RJ \& Rainey WE. Adrenarche - physiology, biochemistry and human disease. Clinical Endocrinology 200460 288-296. (https://doi. org/10.1046/j.1365-2265.2003.01858.x)

4 Auchus RJ. The classic and nonclassic concenital adrenal hyperplasias. Endocrine Practice 201521 383-389. (https://doi. org/10.4158/EP14474.RA)

5 Sharifi N, Gulley JL \& Dahut WL. An update on androgen deprivation therapy for prostate cancer. Endocrine-Related Cancer 2010 17 R305-R315. (https://doi.org/10.1677/ERC-10-0187)

6 Sharifi N. Mechanisms of androgen receptor activation in castrationresistant prostate cancer. Endocrinology 2013154 4010-4017. (https:// doi.org/10.1210/en.2013-1466)

7 Speiser PW, Arlt W, Auchus RJ, Baskin LS, Conway GS, Merke DP, Meyer-Bahlburg HFL, Miller WL, Murad MH, Oberfield SE et al. Congenital adrenal hyperplasia due to steroid 21-hydroxylase deficiency: an Endocrine Society clinical practice guideline. Journal of Clinical Endocrinology and Metabolism 2018103 4043-4088. (https:// doi.org/10.1210/jc.2018-01865)
8 Auchus RJ \& Sharifi N. Sex hormones and prostate cancer. Annual Review of Medicine 202071 33-45. (https://doi.org/10.1146/annurevmed-051418-060357)

9 de Bono JS, Logothetis CJ, Molina A, Fizazi K, North S, Chu L, Chi KN, Jones RJ, Goodman Jr OB, Saad F et al. Abiraterone and increased survival in metastatic prostate cancer. New England Journal of Medicine 2011364 1995-2005. (https://doi.org/10.1056/ NEJMoa1014618)

10 Ryan CJ, Smith MR, de Bono JS, Molina A, Logothetis CJ, de Souza P, Fizazi K, Mainwaring P, Piulats JM, Ng S et al. Abiraterone in metastatic prostate cancer without previous chemotherapy. New England Journal of Medicine 2013368 138-148. (https://doi. org/10.1056/NEJMoa1209096)

11 Fizazi K, Tran N, Fein L, Matsubara N, Rodriguez-Antolin A, Alekseev BY, Ozguroglu M, Ye D, Feyerabend S, Protheroe A et al. Abiraterone plus prednisone in metastatic, castration-sensitive prostate cancer. New England Journal of Medicine 2017377 352-360. (https://doi.org/10.1056/NEJMoa1704174)

12 James ND, de Bono JS, Spears MR, Clarke NW, Mason MD, Dearnaley DP, Ritchie AWS, Amos CL, Gilson C, Jones RJ et al. Abiraterone for prostate cancer not previously treated with hormone therapy. New England Journal of Medicine 2017377 338-351. (https:// doi.org/10.1056/NEJMoa1702900)

13 Attard G, Reid AH, Yap TA, Raynaud F, Dowsett M, Settatree S, Barrett M, Parker C, Martins V, Folkerd E et al. Phase I clinical trial of a selective inhibitor of CYP17, abiraterone acetate, confirms that castration-resistant prostate cancer commonly remains hormone driven. Journal of Clinical Oncology 200826 4563-4571. (https://doi. org/10.1200/JCO.2007.15.9749)

14 Auchus RJ, Buschur EO, Chang AY, Hammer GD, Ramm C, Madrigal D, Wang G, Gonzalez M, Xu XS, Smit JW et al. Abiraterone acetate to lower androgens in women with classic 21-hydroxylase deficiency. Journal of Clinical Endocrinology and Metabolism 201499 2763-2770. (https://doi.org/10.1210/jc.2014-1258)

15 Rege J, Turcu AF, Kasa-Vubu JZ, Lerario AM, Auchus GC, Auchus RJ, Smith JM, White PC \& Rainey WE. 11-Ketotestosterone is the dominant circulating bioactive androgen during normal and premature adrenarche. Journal of Clinical Endocrinology and Metabolism 2018103 4589-4598. (https://doi.org/10.1210/jc.2018-00736)

16 Turcu AF \& Auchus RJ. Clinical significance of 11-oxygenated androgens. Current Opinion in Endocrinology, Diabetes, and Obesity 201724 252-259. (https://doi.org/10.1097/MED.0000000000000334)

17 Turcu AF, Nanba AT, Chomic R, Upadhyay SK, Giordano TJ, Shields JJ, Merke DP, Rainey WE \& Auchus RJ. Adrenal-derived 11-oxygenated 19-carbon steroids are the dominant androgens in classic 21-hydroxylase deficiency. European Journal of Endocrinology 2016174 601-609. (https://doi.org/10.1530/EJE-15-1181)

18 Nanba AT, Rege J, Ren J, Auchus RJ, Rainey WE \& Turcu AF. 11-Oxygenated C19 steroids do not decline With age in women. Journal of Clinical Endocrinology and Metabolism 2019104 2615-2622. (https://doi.org/10.1210/jc.2018-02527)

19 O'Reilly MW, Kempegowda P, Jenkinson C, Taylor AE, Quanson JL, Storbeck KH \& Arlt W. 11-Oxygenated C19 steroids are the predominant androgens in polycystic ovary syndrome. Journal of Clinical Endocrinology and Metabolism 2017102 840-848. (https://doi. org/10.1210/jc.2016-3285)

20 Pretorius E, Africander DJ, Vlok M, Perkins MS, Quanson J \& Storbeck KH. 11-Ketotestosterone and 11-ketodihydrotestosterone in castration resistant prostate cancer: potent androgens which can no longer be ignored. PLoS ONE 201611 e0159867. (https://doi. org/10.1371/journal.pone.0159867)

21 Attard G, Reid AHM, Auchus RJ, Hughes BA, Cassidy AM, Thompson E, Oommen NB, Folkerd E, Dowsett M, Arlt W et al. Clinical and biochemical consequences of CYP17A1 inhibition with abiraterone given with and without exogenous glucocorticoids in castrate men with advanced prostate cancer. Journal of Clinical 
Endocrinology and Metabolism 201297 507-516. (https://doi. org/10.1210/jc.2011-2189)

22 Auchus RJ, Yu MK, Nguyen S \& Mundle SD. Use of prednisone with abiraterone acetate in metastatic castration-resistant prostate cancer. Oncologist 201419 1231-1240. (https://doi.org/10.1634/ theoncologist.2014-0167)

23 Gomes LG, Huang N, Agrawal V, Mendonça BB, Bachega TA \& Miller WL. Extraadrenal 21-hydroxylation by CYP2C19 and CYP3A4: effect on 21-hydroxylase deficiency. Journal of Clinical Endocrinology and Metabolism 200994 89-95. (https://doi. org/10.1210/jc.2008-1174)

24 Mallappa A, Sinaii N, Kumar P, Whitaker MJ, Daley LA, Digweed D, Eckland DJ, Van Ryzin C, Nieman LK, Arlt W et al. A phase 2 study of Chronocort, a modified-release formulation of hydrocortisone, in the treatment of adults with classic congenital adrenal hyperplasia. Journal of Clinical Endocrinology and Metabolism 2015100 1137-1145. (https://doi.org/10.1210/jc.2014-3809)

25 Nella AA, Mallappa A, Perritt AF, Gounden V, Kumar P, Sinaii N, Daley LA, Ling A, Liu CY, Soldin SJ et al. A Phase 2 study of continuous subcutaneous hydrocortisone infusion in adults with congenital adrenal hyperplasia. Journal of Clinical Endocrinology and Metabolism 2016101 4690-4698. (https://doi.org/10.1210/jc.20161916)

26 Turcu AF, Spencer-Segal JL, Farber RH, Luo R, Grigoriadis DE, Ramm CA, Madrigal D, Muth T, O'Brien CF \& Auchus RJ. Singledose study of a corticotropin-releasing factor receptor-1 antagonist in women with 21-hydroxylase deficiency. Journal of Clinical Endocrinology and Metabolism 2016101 1174-1180. (https://doi. org/10.1210/jc.2015-3574)

27 Garrido M, Peng HM, Yoshimoto FK, Upadhyay SK, Bratoeff E \& Auchus RJ. A-ring modified steroidal azoles retain similar potent and slowly reversible CYP17A1 inhibition as abiraterone. Journal of Steroid Biochemistry and Molecular Biology 2014143 1-10. (https://doi. org/10.1016/j.jsbmb.2014.01.013)
28 Barnard M, Quanson JL, Mostaghel E, Pretorius E, Snoep JL \& Storbeck KH. 11-Oxygenated androgen precursors are the preferred substrates for aldo-keto reductase 1C3 (AKR1C3): implications for castration resistant prostate cancer. Journal of Steroid Biochemistry and Molecular Biology 2018183 192-201. (https://doi.org/10.1016/j. jsbmb.2018.06.013)

29 du Toit T, Bloem LM, Quanson JL, Ehlers R, Serafin AM \& Swart AC. Profiling adrenal 11 $\beta$-hydroxyandrostenedione metabolites in prostate cancer cells, tissue and plasma: UPC(2)-MS/MS quantification of 11ß-hydroxytestosterone, 11keto-testosterone and 11keto-dihydrotestosterone. Journal of Steroid Biochemistry and Molecular Biology 2017166 54-67. (https://doi.org/10.1016/j. jsbmb.2016.06.009)

30 Gent R, du Toit T, Bloem LM \& Swart AC. 11ß-Hydroxysteroid dehydrogenase isoforms: pivotal catalytic activities yield potent C11-oxy C19 steroids with 11ßHSD2 favouring 11-ketotestosterone. Journal of Steroid Biochemistry and Molecular Biology 2019189 116-126. (https://doi.org/10.1016/10.1016/j.jsbmb.2019.02.013)

31 Casey ML, Winkel CA \& MacDonald PC. Conversion of progesterone to deoxycorticosterone in the human fetus: steroid 21-hydroxylase activity in fetal tissues. Journal of Steroid Biochemistry 198318 449-452. (https://doi.org/10.1016/0022-4731(83)90064-x)

32 Costa-Barbosa FA, Carvalho VM, Nakamura OH, Bachega TA, Vieira JG \& Kater CE. Zona fasciculata 21-hydroxysteroids and precursor-to-product ratios in 21-hydroxylase deficiency: further characterization of classic and non-classic patients and heterozygote carriers. Journal of Endocrinological Investigation 201134 587-592. (https://doi.org/10.3275/7273)

33 Yoshimoto FK, Zhou Y, Peng HM, Stidd D, Yoshimoto JA, Sharma KK, Matthew S \& Auchus RJ. Minor activities and transition state properties of the human steroid hydroxylases cytochromes P450c17 and P450c21, from reactions observed with deuteriumlabeled substrates. Biochemistry 201251 7064-7077. (https://doi. org/10.1021/bi300895w)

Received 11 November 2019

Revised version received 30 January 2020

Accepted 10 February 2020 